\title{
Optimization of Balking and Reneging Queue with Vacation Interruption under N-Policy
}

\author{
P. Vijaya Laxmi, ${ }^{1}$ V. Goswami, ${ }^{2}$ and K. Jyothsna ${ }^{1}$ \\ ${ }^{1}$ Department of Applied Mathematics, Andhra University, Visakhapatnam 530 003, India \\ ${ }^{2}$ School of Computer Application, KIIT University, Bhubaneswar 751 024, India \\ Correspondence should be addressed to P. Vijaya Laxmi; vijaya_iit2003@yahoo.co.in
}

Received 4 February 2013; Accepted 14 June 2013

Academic Editor: Zhihua Cui

Copyright (c) 2013 P. Vijaya Laxmi et al. This is an open access article distributed under the Creative Commons Attribution License, which permits unrestricted use, distribution, and reproduction in any medium, provided the original work is properly cited.

\begin{abstract}
This paper analyzes a finite buffer multiple working vacations queue with balking, reneging, and vacation interruption under $N$ policy. In the working vacation, a customer is served at a lower rate and at the instants of a service completion; if there are at least $N$ customers in the queue, the vacation is interrupted and the server switches to regular busy period otherwise continues the vacation. Using Markov process and recursive technique, we derive the stationary system length distributions at arbitrary epoch. Various performance measures and some special models of the system are presented. Cost analysis is carried out using particle swarm optimization and quadratic fit search method. Finally, some numerical results showing the effect of model parameters on key performance measures of the system are presented.
\end{abstract}

\section{Introduction}

Performance modeling of queueing systems with balking and reneging has attracted many researchers owing to their wide applications in real life congestion problems such as impatient telephone switchboard customers, hospital emergency rooms handling critical patients and perishable goods storage in inventory systems. Balking and reneging are a common phenomena in queues; as a consequence, the customers either decide not to join the queue or depart after joining the queue without getting service due to impatience. Modeling balking and reneging is worthwhile because one obtains new managerial insights. The lost revenues due to balking and reneging in various industries can be enormous. While making decision for the number of servers needed in the service system to meet time-varying demand, the balking and reneging probabilities can be used to estimate the amount of lost business in more practical consideration for the managers as given in Liao [1].

Impatience is the most prominent characteristic as individuals always feel anxious and impatient during waiting for service in real life. The customer's impatient acts should be involved in the study of queueing system to model real situations exactly. Intermittent operation of a service can be economically appealing whenever full time service would result in significant server idle time or would preclude the use of the server in some other productive capacity. On the other hand, having the server inoperative for periods of time may increase the probability of customer losses due to balking and reneging. An $M / M / 1$ queue with customers balking and reneging has been discussed in Haight [2] and Haight [3], respectively. The combined impact of balking and reneging with finite capacity in an $M / M / 1$ queue has been studied by Ancker and Gafarian [4,5]. Abou-ElAta [6] discussed the finite buffer single server queueing system with balking and reneging. Analytical solutions of the single server Markovian overflow queue with balking, reneging and an additional server for longer queue were discussed in Abou-El-Ata and Shawky [7]. A computational algorithm and parameter optimization for a multiserver queue with unreliable server and impatient customers have been discussed by Chia and Jau-Chaun [8]. Choudhury and Medhi [9] presented some aspects of balking and reneging in finite buffer queue. Recently, Rakesh and Sumeet [10] studied an $M / M / 1 / N$ queueing model with retention of reneged customers and balking. 
Queueing systems with server vacations have been studied due to their wide applications in several areas including computer and communication systems, manufacturing and production systems. Queueing systems with server vacations have been studied extensively in Tian and Zhang [11], Ke et al. [12], and so forth. Yue et al. [13] have analyzed an $M / M / 1 / N$ queue with balking, reneging, and server vacations. Vikas and Deepali [14] studied the state dependent bulk service queue with balking, reneging, and server vacation. However, there are numerous situations where the server remains active during the vacation period. Under working vacation $(W V)$ policy, the server can provide service at a lower speed during vacation period rather than stopping service completely. At the end of a vacation if the queue is nonempty, a service period begins with normal service rate; otherwise the server takes another vacation. This policy is called multiple working vacations $(M W V s)$ as introduced by Servi and Finn [15]. Recently, Vijaya Laxmi et al. [16] analyzed an $M / M / 1 / N$ working vacations queue with balking and reneging.

In order to utilize the server effectively, vacation interruption has become an important aspect, wherein the server interrupts the vacation once some indices of the system, such as the number of waiting customers, achieve a certain value in the vacation period. Li and Tian [17] considered a working vacation $M / M / 1$ queue with vacation interruptions. Baba [18] studied an $M / P H / 1$ queue with working vacations and vacation interruptions. Zhang and Hou [19] studied the more general $M / G / 1$ queue with working vacations and vacation interruptions. An $M / M / 1 / M W V$ queue with $N$-policy has been studied in Zhang and $\mathrm{Xu}$ [20].

Queueing models with $N$-policy consider the most common issue of controlling arrivals and reducing the set up costs. The closed-form results in case of impatient customers with working vacations, vacation interruptions under $N$ policy are still not available. This motivated us to study an $N$ policy vacation interruptions queue with balking and reneging. This paper also discusses cost optimization problem under a given cost structure using particle swarm optimization (PSO) and quadratic fit search method (QFSM). PSO is a parallel evolutionary computation technique developed by Kennedy and Eberhart [21] based on the social behavior of animals such as bird flocking, fish schooling, and swarm theory. In the basic PSO algorithm the particle velocities build up too fast, and the optimum of the objective function is skipped. Hence, an inertia term introduced by Shi and Eberhart [22] is added to reduce the velocity. PSO can be easily implemented and is computationally inexpensive. An analysis of the PSO algorithm has been given in Qinghai [23] and Rao [24]. QFSM uses a 3-point pattern for fitting a quadratic function that has a unique optimum, see Rardin [25].

This paper deals with a finite buffer single server queue wherein customers may either balk or renege and the server takes multiple working vacations with a provision for vacation interruption. The server interrupts the vacation when the queue size reaches $N$; otherwise the vacation continues. The steady-state probabilities are obtained at arbitrary epoch through recursive technique which is easy to implement. We formulate a cost model to determine the optimum service rate during normal busy period and during working vacation period using PSO and QFSM.

The rest of the paper is organized as follows. Section 2 presents the description of the model and the steady-state probabilities. Sections 3 and 4 present some special cases, various performance measures, and cost analysis, respectively. Numerical results in the form of tables and graphs are presented in Section 5. Section 6 concludes the paper.

\section{Model Description and Steady-State Probabilities}

We consider a multiple working vacations $M / M / 1 / K$ queueing system with vacation interruptions wherein customers either balk or renege. The following notations and assumptions have been used throughout the paper.

(i) Customers arrive one at a time according to Poisson process with arrival rate $\lambda$. On arrival a customer either decides to join the queue or balk. Let $b_{i}$ represent the probability with which the customer either decides to join the queue or balk with probability $1-b_{i}$, when there are $i$ customers ahead of him in the system. Furthermore, we assume that $0 \leq b_{i+1} \leq b_{i}<$ $1,1 \leq i \leq K-1, b_{0}=1$, and $b_{K}=0$.

(ii) After joining the queue each customer will wait a certain length of time, say $T$, for service to begin before they get impatient and leave the queue without receiving service. This time $T$ is assumed to follow exponential distribution with mean $1 / \alpha$. Since the arrival and departure of an impatient customer without receiving service are independent, the average rate of reneging of a customer is given by $(i-1) \alpha$. Hence, the function of customer's average reneging rate is given by $r_{i}=(i-1) \alpha, 1 \leq i \leq K ; r_{i}=0, i>K$.

(iii) The customers are served on a first-come first-served (FCFS) queue discipline. The service times in normal busy period are assumed to follow exponential distribution with mean $1 / \mu$.

(iv) The server takes working vacations whenever the system becomes empty. At a service completion epoch during vacation, if there are at least $N$ customers waiting in the system, the server ends the vacation and switches to normal service; that is, a vacation interruption occurs; otherwise, it continues the vacation. Furthermore, at vacation termination epoch, if there are less than $N$ customers in the queue, the server begins another vacation; else, it switches to the normal busy period.

(v) During any working vacation the server will serve customers at a rate different (generally lower) from the normal service rate $\mu$. The vacation duration and the service times in vacation period are also assumed 
be exponentially distributed with mean $1 / \phi$ and $1 / \eta$, respectively.

(vi) The interarrival times, vacation times, service times during normal busy period, and service times during vacation are mutually independent.

2.1. Steady-State Equations. At steady-state, let $P_{0, i}, 0 \leq i \leq K$, be the probability that there are $i$ customers in the system when the server is in working vacation and let $P_{1, i}, 1 \leq i \leq K$, be the probability that there are $i$ customers in the system when the server is in normal busy period. Using the Markov theory, the following set of steady-state equations may be written

$$
\begin{gathered}
\lambda P_{0,0}=\eta P_{0,1}+\mu P_{1,1}, \\
\left(\lambda b_{i}+\eta+(i-1) \alpha\right) P_{0, i} \\
=(\eta+i \alpha) P_{0, i+1}+\lambda b_{i-1} P_{0, i-1}, \quad 1 \leq i \leq N-1, \\
\left(\lambda b_{i}+\eta+(i-1) \alpha+\phi\right) P_{0, i} \\
=\lambda b_{i-1} P_{0, i-1}+i \alpha P_{0, i+1}, \quad N \leq i \leq K-1, \\
(\eta+(K-1) \alpha+\phi) P_{0, K}=\lambda b_{K-1} P_{0, K-1}, \\
\left(\mu+\lambda b_{i}+(i-1) \alpha\right) P_{1, i} \\
=\lambda b_{i-1} P_{1, i-1}+(\mu+i \alpha) P_{1, i+1}, \quad 1 \leq i \leq N-1, \\
\left(\mu+\lambda b_{i}+(i-1) \alpha\right) P_{1, i} \\
=\lambda b_{i-1} P_{1, i-1}+(\mu+i \alpha) P_{1, i+1} \\
+\eta P_{0, i+1}+\phi P_{0, i}, \quad N \leq i \leq K-1, \\
(\mu+(K-1) \alpha) P_{1, K}=\lambda b_{K-1} P_{1, K-1}+\phi P_{0, K} .
\end{gathered}
$$

The steady-state probabilities $P_{0, i}\left(P_{1, i}\right)$ can be obtained by solving the previous system of equations recursively that is presented as a theorem later.

Theorem 1. The steady-state probabilities are given by

$$
\begin{array}{cl}
P_{0, i}=\frac{\zeta_{i}}{\sum_{m=0}^{K} \zeta_{m}+\sum_{m=1}^{K} \xi_{m}}, & 0 \leq i \leq K, \\
P_{1, i}=\frac{\xi_{i}}{\sum_{m=0}^{K} \zeta_{m}+\sum_{m=1}^{K} \xi_{m}}, & 1 \leq i \leq K,
\end{array}
$$

where

$$
\begin{array}{r}
\zeta_{K}=1, \quad \zeta_{K-1}=\frac{\phi+\eta+(K-1) \alpha}{\lambda b_{K-1}}, \\
\zeta_{i}=\frac{\left(\lambda b_{i+1}+\eta+\phi+i \alpha\right) \zeta_{i+1}-(i+1) \alpha \zeta_{i+2}}{\lambda b_{i}}, \\
i=K-2, K-3, \ldots, N-1,
\end{array}
$$

$$
\begin{gathered}
\zeta_{i}=\frac{\left(\lambda b_{i+1}+\eta+i \alpha\right) \zeta_{i+1}-(\eta+(i+1) \alpha) \zeta_{i+2},}{\lambda b_{i}}, \\
i=N-2, N-3, \ldots, 0, \\
\xi_{0}=0, \quad \xi_{1}=\frac{\lambda \zeta_{0}-\eta \zeta_{1}}{\mu}, \\
\xi_{i}=\frac{\left(\mu+\lambda b_{i-1}+(i-2) \alpha\right) \xi_{i-1}-\lambda b_{i-2} \xi_{i-2}}{\mu+(i-1) \alpha}, \\
i=2,3, \ldots, N, \\
\xi_{i}=\frac{\left(\mu+\lambda b_{i-1}+(i-2) \alpha\right) \xi_{i-1}-\lambda b_{i-2} \xi_{i-2}-\eta \zeta_{i}-\phi \zeta_{i-1}}{\mu+(i-1) \alpha}, \\
i=N+1, N+2, \ldots, K .
\end{gathered}
$$

Proof. Solving the system of (1) to (6) recursively and using the normalization condition $\sum_{i=0}^{K} P_{0, i}+\sum_{i=1}^{K} P_{1, i}=1$, we obtain the results of the theorem.

\section{Special Cases}

In this section, results pertaining to some models have been deduced from our model by taking specific values for the parameters $\alpha, \eta, b_{i}$, and $N$.

Case $1\left(\alpha \rightarrow 0, b_{i}=1,0 \leq i \leq K, N=1\right)$. The model reduces to $M / M / 1 / K$ queue with multiple working vacations and vacation interruption. We can obtain the steady-state probabilities $P_{0, i}(0 \leq i \leq K)$ and $P_{1, i}(1 \leq i \leq K)$ from (8)-(9), where

$$
\begin{gathered}
\zeta_{K}=1, \quad \zeta_{K-1}=\frac{\phi+\eta}{\lambda}, \quad \zeta_{i}=\frac{(\lambda+\eta+\phi)}{\lambda} \zeta_{i+1}, \\
i=K-2, K-3, \ldots, 0, \\
\xi_{0}=0, \quad \xi_{1}=\frac{\lambda \zeta_{0}-\eta \zeta_{1}}{\mu}, \\
\xi_{i}=\frac{(\mu+\lambda) \xi_{i-1}-\lambda \xi_{i-2}-\eta \zeta_{i}-\phi \zeta_{i-1}}{\mu}, \\
i=2,3, \ldots, K,
\end{gathered}
$$

which match with the results of $M / M / 1 / \infty$ queue with multiple working vacations and vacation interruption $\mathrm{Li}$ and Tian [17] for large $K$ values.

Case $2(N=1$ and without vacation interruptions). The model reduces to $M / M / 1 / K$ queue with balking, reneging and multiple working vacations and results match numerically with the results available in Vijaya Laxmi et al. [16].

Case $3(N=1, \eta \rightarrow 0)$. The model reduces to $M / M / 1 / K$ queue with balking, reneging and multiple vacations. Taking 
the average reneging rate as $r_{i}=(i-n) \alpha, n=0$ in vacation period and $n=1$ in normal busy period, we can obtain the steady-state probabilities $P_{0, i}$ and $P_{1, i}$ from (8)-(9), where

$$
\begin{aligned}
& \zeta_{K}=1, \quad \zeta_{K-1}=\frac{K \alpha+\phi}{\lambda b_{K-1}}, \\
& \xi_{0}=0, \quad \xi_{1}=\frac{\lambda \zeta_{0}-\alpha \zeta_{1}}{\mu}, \\
& \zeta_{i}=\frac{\left(\lambda b_{i+1}+\phi+(i+1) \alpha\right) \zeta_{i+1}-(i+2) \alpha \zeta_{i+2}}{\lambda b_{i}}, \\
& i=K-2, K-3, \ldots, 0, \\
& \xi_{i}=\frac{\left(\mu+\lambda b_{i-1}+(i-2) \alpha\right) \xi_{i-1}-\lambda b_{i-2} \xi_{i-2}-\phi \zeta_{i-1}}{\mu+(i-1) \alpha \quad i=2,3, \ldots, K .}
\end{aligned}
$$

The previous results match numerically with the results of Yue et al. [13].

Case $4(\eta \rightarrow 0$ and $\phi \rightarrow \infty)$. The model reduces to $M /$ $M / 1 / K$ queue with balking and reneging. These results match with the results available in the literature.

\section{Performance Measures}

Once the steady-state probabilities are obtained, one can evaluate various performance measures of the model like probability that the server is in normal busy period $\left(P_{b}\right)$, probability that the server is in working vacation $\left(P_{w v}\right)$, average queue length $\left(L_{q}\right)$, average system length $\left(L_{s}\right)$, and they are given by

$$
\begin{aligned}
& P_{b}=\sum_{i=1}^{K} P_{1, i}, \quad P_{w v}=\sum_{i=0}^{K} P_{0, i}, \\
& L_{q}=\sum_{i=1}^{K}(i-1)\left(P_{0, i}+P_{1, i}\right), \quad L_{s}=\sum_{i=1}^{K} i\left(P_{0, i}+P_{1, i}\right) .
\end{aligned}
$$

When a customer finds $i$ customers in the system upon his arrival, then the instantaneous balking rate is $\lambda\left(1-b_{i}\right)$, as the probability that a customer balks in the system is $1-b_{i}$. Using the concepts of Ancker and Gafarian [4, 5], we can obtain the average balking rate (B.R.) as

$$
\text { B.R. }=\sum_{i=1}^{K} \lambda\left(1-b_{i}\right) P_{0, i}+\sum_{i=1}^{K} \lambda\left(1-b_{i}\right) P_{1, i} .
$$

If there are $i$ customers in the system and server is available, then there are $(i-1)$ waiting customers in the queue. Since any one of the $(i-1)$ customers in the queue may renege, the instantaneous reneging rate is $(i-1) \alpha$. Again, following the models of Ancker and Gafarian $[4,5]$, the average reneging rate (R.R.) is given by

$$
\text { R.R. }=\sum_{i=1}^{K}(i-1) \alpha P_{0, i}+\sum_{i=1}^{K}(i-1) \alpha P_{1, i} .
$$

The average rate of customer loss (L.R.) is the sum of the average balking rate and the average reneging rate. Thus, we have

$$
\text { L.R. }=\text { B.R. + R.R. }
$$

4.1. Cost Model. In practice, queueing managers are always interested in minimizing operating cost of unit time. In this section, we first develop a steady-state expected cost function per unit time, in which either service rate during normal busy period or service rate during working vacation period is the decision variable. Our objective is to determine the optimum value of $\mu$ or $\eta$ to minimize the cost function.

Let us define the following cost elements:

$C_{\mu} \equiv$ service cost per unit time during normal busy period,

$C_{\eta} \equiv$ service cost per unit time during working vacation period,

$C_{l q} \equiv$ cost per unit time when a customer joins the queue and waits for service,

$C_{l r} \equiv$ cost per unit time when a customer balks or reneges.

The cost parameters are assumed to be linear in the average number of the indicated quantities such as the average queue length $\left(L_{q}\right)$ and the average rate of customer loss (L.R.). Based on the definitions of each cost element listed previously and its corresponding system performance measures, the cost minimization problem can be illustrated mathematically as:

$$
\text { Minimize : } f(x)=C_{\mu} \mu+C_{\eta} \eta+C_{l q} L_{q}+C_{l r} \text { L.R., }
$$

where $x$ can be either service rate during normal busy period $(\mu)$ or service rate during working vacation period $(\eta)$. The objective is to determine the optimal service rate $\mu^{*}$ or $\eta^{*}$ to minimize the cost function $f$. It is a difficult task to develop analytic results for the optimum value of $\mu$ or $\eta$, because the expected cost function is highly complex. We note that the derivatives of the operating cost function per unit time are not easily available. We solve the above stated optimization problem using PSO and QFSM. Both methods give identical results as shown in the next section.

\subsubsection{Algorithm for PSO}

Step 1. Initialize a population of particles of size $m$ with random positions in the problem space. For each particle, evaluate the objective function value.

Step 2. As all the particles will be moving to the optimal point with a velocity, find the velocities of particles. Initially, all particle velocities are assumed to be zero.

Step 3. Store each particle's coordinates associated with the best solution in pbest and the coordinates of the particle associated with the overall best solution in $g$ best. 
TABLE 1: Optimum values for various values of $\eta$ and $\lambda$.

\begin{tabular}{|c|c|c|c|c|c|c|c|c|}
\hline \multirow{2}{*}{$\eta$} & \multicolumn{4}{|c|}{$\lambda=2.0$} & \multicolumn{4}{|c|}{$\lambda=2.2$} \\
\hline & $\mu^{*}$ & $f^{*}$ & $L_{q}^{*}$ & $L . R^{*}$ & $\mu^{*}$ & $f^{*}$ & $L_{q}^{*}$ & $L . R .^{*}$ \\
\hline 1.5 & 2.01604 & 104.092 & 1.59964 & 0.53122 & 2.23676 & 112.397 & 1.75077 & 0.58658 \\
\hline 1.7 & 2.00395 & 104.058 & 1.50002 & 0.49868 & 2.23122 & 112.527 & 1.65287 & 0.55431 \\
\hline 1.9 & 1.98578 & 104.060 & 1.40553 & 0.46780 & 2.21962 & 112.663 & 1.55904 & 0.52338 \\
\hline 2.1 & 1.96241 & 104.125 & 1.31656 & 0.43871 & 2.20261 & 112.828 & 1.46968 & 0.49392 \\
\hline 2.3 & 1.93473 & 104.274 & 1.23334 & 0.41148 & 2.18085 & 113.043 & 1.38511 & 0.46603 \\
\hline
\end{tabular}

TABLE 2: $\eta^{*}$ from PSO and QFSM.

\begin{tabular}{cccccr}
\hline$\mu$ & \multicolumn{2}{c}{ PSO } & & & QFSM \\
& $\eta^{*}$ & & $f^{*}$ & Initial 3-point pattern & $\eta^{*}$ \\
\hline 2.3 & 1.51326 & 97.8642 & $(1.4,1.5,1.6)$ & 1.51326 & 97.8642 \\
2.4 & 1.45479 & 98.7762 & $(1.4,1.5,1.6)$ & 1.45479 & 98.7762 \\
2.5 & 1.40225 & & $(1.3,1.4,1.5)$ & 1.40225 & 99.7727 \\
2.6 & 1.35504 & & $(1.3,1.4,1.5)$ & 1.35504 & 100.843 \\
2.7 & 1.31262 & 100.84727 & $(1.2,1.3,1.4)$ & 1.31262 & 101.979 \\
\hline
\end{tabular}

Step 4. Compare each particle's fitness with particle's pbest. If current value is better, then set pbest value to the current value. Compare each particle's fitness with the population's overall previous best (gbest), and if any particle's current value is better than $g$ best, then set $g$ best to the current value.

Step 5. Change the velocity $v_{j}(i)$ and position $x_{j}(i)$ of the $j$ th particle in the $i$ th iteration according to the following equations, respectively,

$$
\begin{gathered}
v_{j}(i)=\varphi(i) v_{j}(i-1)+c_{1} \operatorname{rand}(\cdot)\left(p \operatorname{best}(j)-x_{j}(i-1)\right) \\
+c_{2} \operatorname{rand}(\cdot)\left(g \text { best }-x_{j}(i-1)\right), \quad 1 \leq j \leq m, \\
x_{j}(i)=x_{j}(i-1)+v_{j}(i), \quad 1 \leq j \leq m,
\end{gathered}
$$

where $\operatorname{rand}(\cdot)$ is a random number between $(0,1), c_{1}$ and $c_{2}$ are learning factors, and $\varphi(i)$ is the inertia weight given by

$$
\varphi(i)=\varphi_{\max }-\left(\frac{\varphi_{\max }-\varphi_{\min }}{i_{\max }}\right) i
$$

with $\varphi_{\max }, \varphi_{\min }$ being the maximum and minimum values of the inertia weight $\left(\varphi_{\max }=0.9, \varphi_{\min }=0.4\right.$ are commonly used) and $i_{\max }$ the maximum number of iterations.

Step 6. Loop to Step 3 until the stopping criterion is met. The stopping criterion is that the number of iterations is completed or the difference between two successive gbest values is less than a predefined error tolerance.

4.1.2. Algorithm for QFSM. Given a 3-point pattern, we can fit a quadratic function through corresponding functional values that have a unique minimum, $x^{q}$, for the given objective function $f(x)$. Quadratic fit uses this approximation to improve the current 3-point pattern by replacing one of its points with approximate optimum $x^{q}$. The unique optimum $x^{q}$ of the quadratic function agreeing with $f(x)$ at 3-point operation $\left(x^{l}, x^{m}, x^{h}\right)$ given by Rardin [25] occurs at

$$
\begin{aligned}
x^{q} \cong \frac{1}{2}[ & \left(f\left(x^{l}\right)\left[s^{m}-s^{h}\right]+f\left(x^{m}\right)\left[s^{h}-s^{l}\right]\right. \\
& \left.+f\left(x^{h}\right)\left[s^{l}-s^{m}\right]\right) \\
& \times\left(f\left(x^{l}\right)\left[x^{m}-x^{h}\right]+f\left(x^{m}\right)\left[x^{h}-x^{l}\right]\right. \\
& \left.\left.+f\left(x^{h}\right)\left[x^{l}-x^{m}\right]\right)^{-1}\right],
\end{aligned}
$$

where $s^{l}=\left(x^{l}\right)^{2}, s^{m}=\left(x^{m}\right)^{2}, s^{h}=\left(x^{h}\right)^{2}$.

\section{Numerical Discussions}

In this section, we illustrate the results obtained in the previous sections numerically and discuss the effect of system parameters on the performance indices. The differences of parameters, such as the lower service rate $(\eta)$, vacation rate $(\phi)$, vacation interruption, and $N$ policy, also influence the various performance measures of the model. We fix the capacity of the system as $K=10$ and $N=4$, and the parameters of the model are assumed as $\lambda=1.8, \mu=2.5$, $\eta=1.9, \phi=1.2$, and $\alpha=0.3$, unless they are considered as variables. The balking function is taken as $b_{i}=1-\left(i / K^{2}\right)$, $1 \leq i \leq K-1, b_{0}=1, b_{K}=0$, and the various cost elements are chosen as $C_{\mu}=18, C_{\eta}=15, C_{l q}=25, C_{l r}=10$. The swarm size, maximum number of iterations, and learning factors of PSO algorithm are taken as 20,50 and $c_{1}=c_{2}=2$, respectively, and the tolerance of QFSM is $\kappa=1 \times 10^{-6}$.

Table 1 presents the optimum values of $\mu$ along with $L_{q}^{*}$ and L.R. ${ }^{*}$ for various $\eta$ and $\lambda$, using particle swarm optimization. For a fixed $\lambda$, decreasing trend is observed in $\mu^{*}, L_{q}^{*}$, and $L . R .^{*}$ with the increase of $\eta$. For a fixed $\eta$, increasing trend is observed in $\mu^{*}, L_{q}^{*}$, and L.R. ${ }^{*}$ with 
TABLE 3: Various performance measures for different balking functions, $\mu, \alpha$, and $\phi$.

\begin{tabular}{|c|c|c|c|c|c|c|}
\hline & & $P_{b}$ & $P_{w v}$ & $L_{q}$ & L.R. & $f$ \\
\hline & $b_{i}=1-\left(i / K^{2}\right)$ & 0.207206 & 0.792794 & 0.943660 & 0.313380 & 100.225 \\
\hline \multirow[t]{3}{*}{$\mu=2.5$} & $b_{i}=1 /(i+1)$ & 0.009290 & 0.990710 & 0.252221 & 0.674025 & 86.5458 \\
\hline & $b_{i}=e^{-i}$ & 0.000502 & 0.999498 & 0.151711 & 0.740087 & 84.6936 \\
\hline & $b_{i}=1-\left(i / K^{2}\right)$ & 0.137534 & 0.862466 & 0.793431 & 0.264904 & 113.985 \\
\hline \multirow[t]{3}{*}{$\mu=3.5$} & $b_{i}=1 /(i+1)$ & 0.006559 & 0.993441 & 0.248745 & 0.671253 & 104.431 \\
\hline & $b_{i}=e^{-i}$ & 0.000362 & 0.999637 & 0.151550 & 0.739925 & 102.688 \\
\hline & $\alpha=0.2$ & 0.252143 & 0.747857 & 1.101570 & 0.254461 & 103.584 \\
\hline \multirow[t]{3}{*}{$\mu=2.5$} & $\alpha=0.5$ & 0.144430 & 0.855570 & 0.746728 & 0.399321 & 96.1614 \\
\hline & $\alpha=0.8$ & 0.088249 & 0.911750 & 0.577965 & 0.484742 & 92.7966 \\
\hline & $\alpha=0.2$ & 0.163313 & 0.836687 & 0.878758 & 0.204519 & 115.514 \\
\hline \multirow[t]{3}{*}{$\mu=3.5$} & $\alpha=0.5$ & 0.099092 & 0.900908 & 0.668530 & 0.358484 & 111.798 \\
\hline & $\alpha=0.8$ & 0.062491 & 0.937509 & 0.542701 & 0.455723 & 109.625 \\
\hline & $\phi=0.9$ & 0.199081 & 0.800919 & 0.951578 & 0.315933 & 100.449 \\
\hline \multirow[t]{3}{*}{$\mu=2.5$} & $\phi=1.1$ & 0.204683 & 0.795317 & 0.946113 & 0.314170 & 100.295 \\
\hline & $\phi=1.4$ & 0.211783 & 0.788217 & 0.939225 & 0.31195 & 100.100 \\
\hline & $\phi=0.9$ & 0.131773 & 0.868227 & 0.807812 & 0.269538 & 114.391 \\
\hline \multirow[t]{2}{*}{$\mu=3.5$} & $\phi=1.1$ & 0.135742 & 0.864258 & 0.797890 & 0.266340 & 114.111 \\
\hline & $\phi=1.4$ & 0.140790 & 0.859209 & 0.785357 & 0.262303 & 113.757 \\
\hline
\end{tabular}

TABLE 4: Various performance measures for different balking functions, $\eta, \alpha$, and $\phi$.

\begin{tabular}{|c|c|c|c|c|c|c|}
\hline & & $P_{b}$ & $P_{w v}$ & $L_{q}$ & L.R. & $f$ \\
\hline \multirow{3}{*}{$\eta=2.1$} & $b_{i}=1-\left(i / K^{2}\right)$ & 0.184638 & 0.815362 & 0.854715 & 0.284490 & 100.713 \\
\hline & $b_{i}=1 /(i+1)$ & 0.007447 & 0.992553 & 0.219831 & 0.626807 & 88.2638 \\
\hline & $b_{i}=e^{-i}$ & 0.000393 & 0.999607 & 0.132791 & 0.692532 & 86.7451 \\
\hline \multirow{3}{*}{$\eta=2.4$} & $b_{i}=1-\left(i / K^{2}\right)$ & 0.154568 & 0.845432 & 0.734459 & 0.245367 & 101.815 \\
\hline & $b_{i}=1 /(i+1)$ & 0.005443 & 0.994557 & 0.181192 & 0.566167 & 91.1915 \\
\hline & $b_{i}=e^{-i}$ & 0.000279 & 0.999720 & 0.110306 & 0.631206 & 90.0697 \\
\hline \multirow{3}{*}{$\eta=2.1$} & $\alpha=0.2$ & 0.226048 & 0.773952 & 1.00062 & 0.231765 & 103.833 \\
\hline & $\alpha=0.5$ & 0.127751 & 0.872249 & 0.674428 & 0.361304 & 96.9737 \\
\hline & $\alpha=0.8$ & 0.077759 & 0.922240 & 0.521827 & 0.438267 & 93.9284 \\
\hline \multirow{3}{*}{$\eta=2.4$} & $\alpha=0.2$ & 0.190649 & 0.809351 & 0.862265 & 0.200605 & 104.563 \\
\hline & $\alpha=0.5$ & 0.106066 & 0.893934 & 0.578489 & 0.310791 & 98.5701 \\
\hline & $\alpha=0.8$ & 0.064377 & 0.935623 & 0.448454 & 0.377461 & 95.9859 \\
\hline \multirow{3}{*}{$\eta=2.1$} & $\phi=0.9$ & 0.176926 & 0.823074 & 0.859298 & 0.285968 & 100.842 \\
\hline & $\phi=1.1$ & 0.182233 & 0.817767 & 0.856141 & 0.284950 & 100.753 \\
\hline & $\phi=1.4$ & 0.189022 & 0.810978 & 0.852124 & 0.283654 & 100.640 \\
\hline \multirow{3}{*}{$\eta=2.4$} & $\phi=0.9$ & 0.147518 & 0.852482 & 0.735379 & 0.245664 & 101.841 \\
\hline & $\phi=1.1$ & 0.152358 & 0.847642 & 0.734747 & 0.245460 & 101.823 \\
\hline & $\phi=1.4$ & 0.158625 & 0.841375 & 0.733932 & 0.245197 & 101.800 \\
\hline
\end{tabular}

the increase of $\lambda$. But the minimum cost increases with the increase of $\eta$ and $\lambda$.

To ensure the reliability of PSO algorithm, we compared our results with QFSM, and the results are shown in Table 2. Though both these methods give identical results, the advantage of using PSO algorithm lies in the ease with which it can be tuned and implemented using only a velocity operator to drive the search throughout the search space, whereas the QFSM is dependent on the proper choice of the initial 3-point approximation. It is also observed that convergence is faster in PSO algorithm.

The effect of $\mu$ and $\eta$ on various performance measures of the model, for various balking functions, $\alpha$ and $\phi$, is presented in Tables 3 and 4 , respectively. Note that for any $\mu(\eta)$, the balking function $b_{i}=1-\left(i / K^{2}\right)$ yields the lowest $L . R$. which supports our choice of the balking function as $b_{i}=1-\left(i / K^{2}\right)$. On the other hand, for any choice of the balking function $P_{b}, L_{q}$, and L.R. decrease with the increase of $\mu(\eta)$, while $P_{w v}$ 


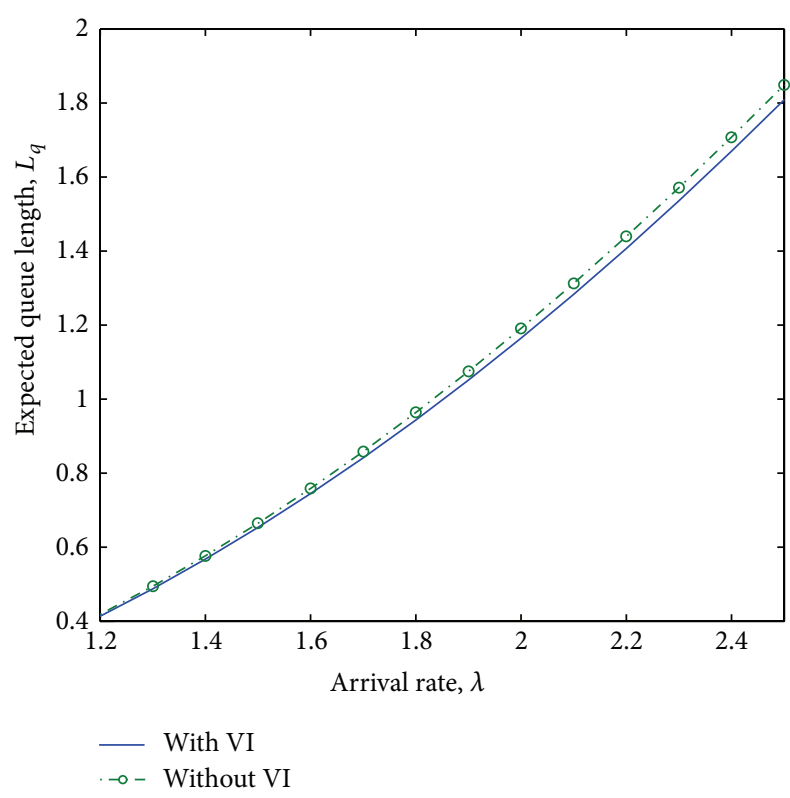

FIGURE 1: Impact of $\lambda$ on $L_{q}$ with and without vacation interruption.

and cost increase with $\mu(\eta)$, as intuitively expected. From the tables it can also be observed that for fixed $\mu(\eta)$, increase in $\alpha$ results in the decrease of $P_{b}, L_{q}$, and cost, whereas the other performance measures $P_{w v}$ and L.R. increase with $\alpha$. Further, for fixed $\alpha, P_{b}, L_{q}$, and $L . R$. decrease with the increase of $\mu(\eta)$. It can also be observed that as $\mu(\eta)$ increases, $P_{b}, L_{q}$, and $L . R$. decrease for any $\phi$. Moreover, for fixed $\mu(\eta)$, except $P_{b}$ all the other performance measures decrease with the increase of $\phi$. This is because as $\phi$ increases, the vacation duration decreases and the server switches to normal busy period during which service is provided with rate $\mu$.

Figure 1 shows the effect of the arrival rate $(\lambda)$ on the expected number of customers in the queue $\left(L_{q}\right)$ with and without vacation interruptions $(V I)$. It can be observed that with the increase of $\lambda$, the expected queue length increases as it should be. Further, one may also observe that for higher values of $\lambda, L_{q}$ is less in the vacation interruptions model as compared to the model without vacation interruptions. This is due to the fact that in vacation interruptions model, the server tends to interrupt the vacation and switches to normal service rate when more customers arrive during vacation. The impact of arrival rate $(\lambda)$ on the expected number of customers in the queue $\left(L_{q}\right)$ with and without balking and reneging $(B \& R)$ is shown in Figure 2. It can be seen that as arrival rate increases $L_{q}$ monotonically increases. Moreover, the model with balking and reneging outperforms the model without balking and reneging.

Figures 3 and 4 plot the effect of the average rate of customer loss (L.R.) for different capacity of the system $(K)$ and threshold value $(N)$, respectively, with and without vacation interruptions. From the figures it can be seen that as the buffer size $(K)$ increases the customer loss decreases, whereas it increases with the increase of $N$, as intuitively expected. Further, in both the figures L.R. is higher in the case of without vacation interruptions as compared to vacation

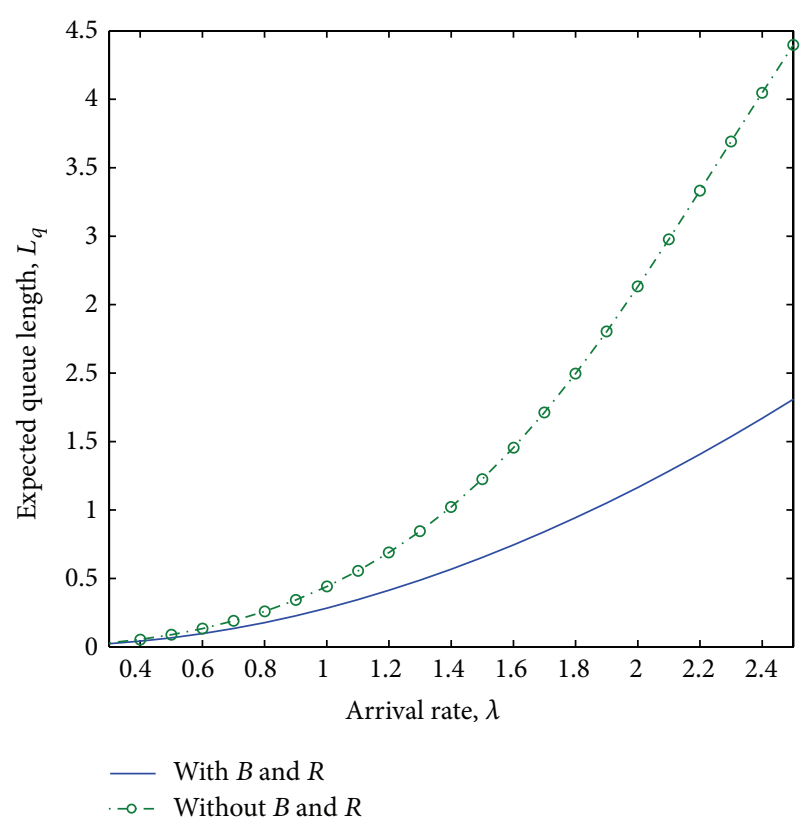

FIGURE $2: \lambda$ versus $L_{q}$ with and without balking and reneging.

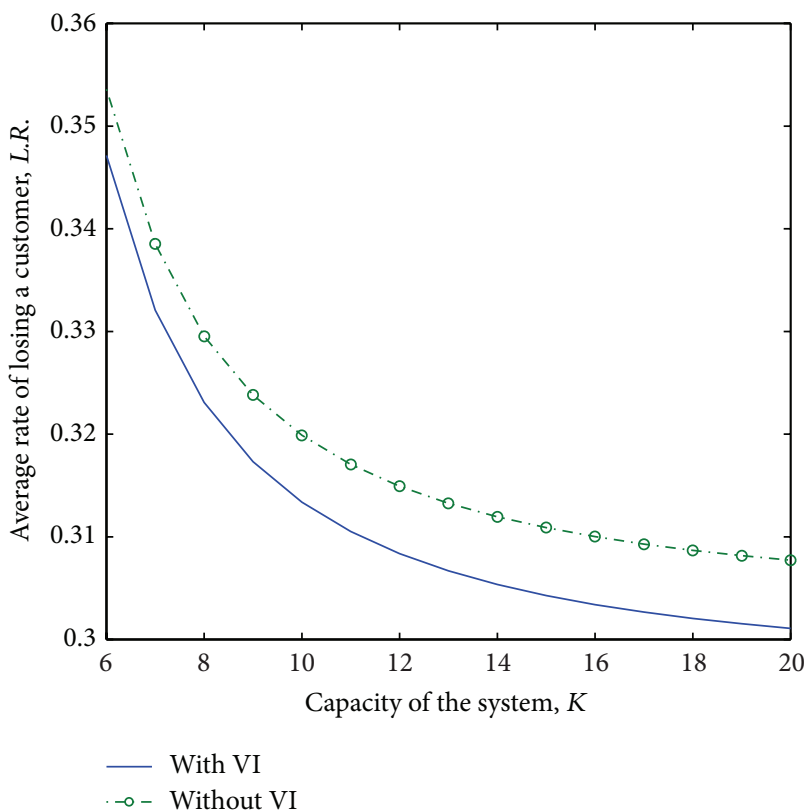

FIGURE 3: Effect of $K$ on $L . R$.

interruptions model implying that a job has to wait more in models without vacation interruptions.

Figure 5 presents the effect of service rate during vacation $(\eta)$ on the average number of customers in the queue $\left(L_{q}\right)$ for various vacation rates $(\phi)$. As expected, $L_{q}$ monotonically decreases as $\eta$ increases for any $\phi$. Further, we observe that for $\eta \leq \mu$, the expected queue lengths decrease with the increase of $\phi$, and when $\eta$ crosses $\mu$, this trend is reversed. This shows that for better performance of the model $\eta$ should be chosen less than $\mu$. Figure 6 depicts the minimum and 


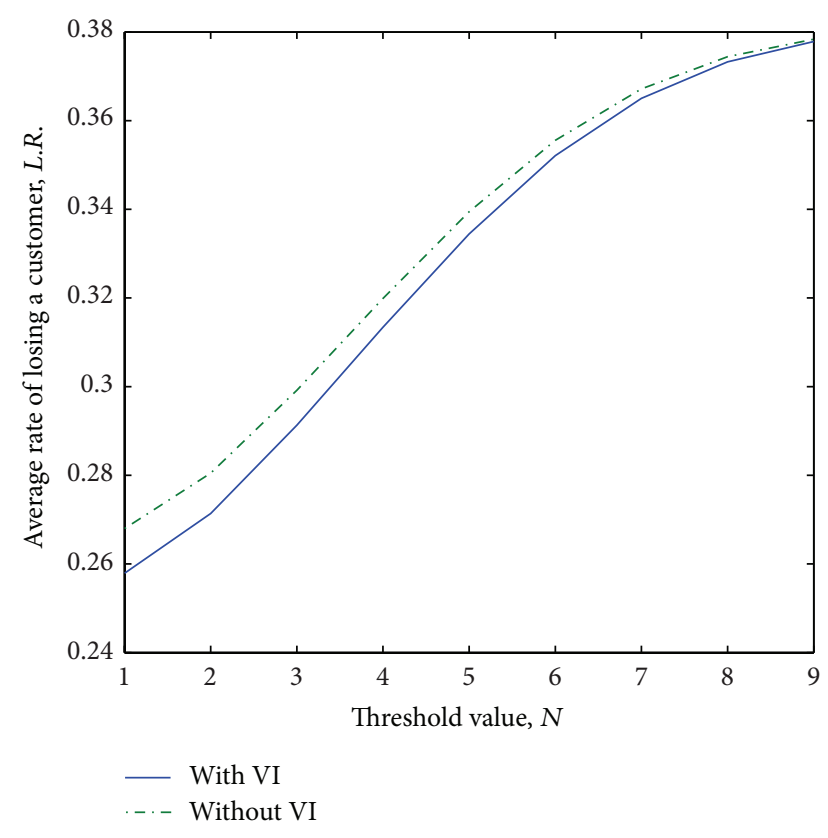

FIGURE 4: Effect of $N$ on L.R.

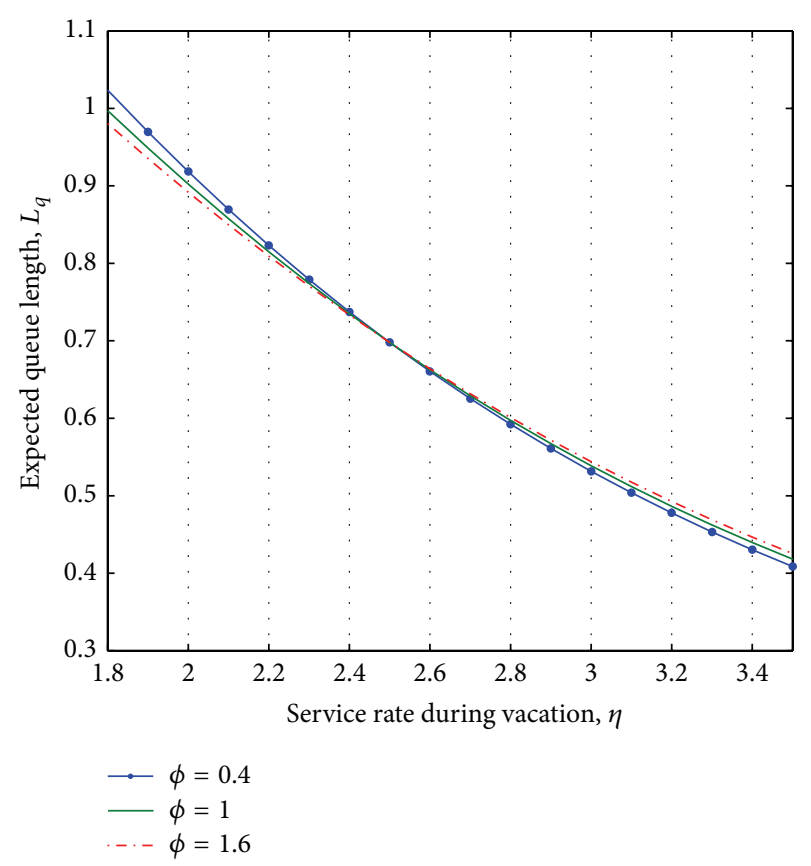

FIGURE 5: Impact of $\eta$ on $L_{q}$.

average costs for number of iterations considered in PSO, when $\eta$ is a decision variable. From the figure, it is observed that though initially the average cost varies widely from iteration to iteration, stability is attained after some iterations.

\section{Conclusions}

In this paper, we have carried out an analysis of a finite-buffer balking and reneging Markovian multiple working vacations queue with vacation interruptions under $N$-policy that have

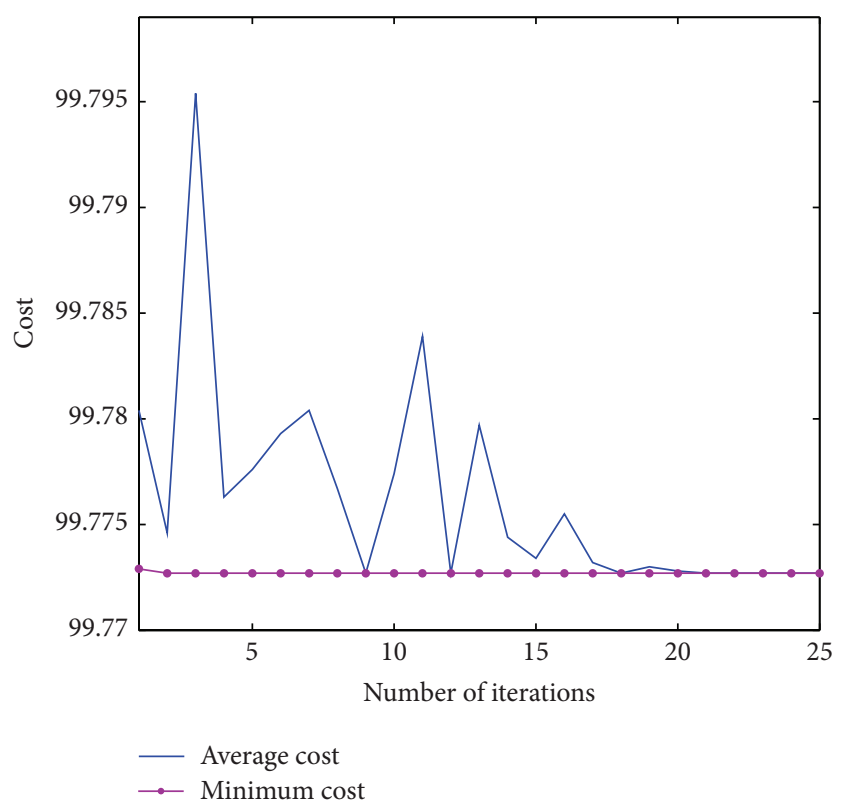

FIGURE 6: Iterations in PSO versus cost.

potential applications in modeling computer and telecommunication systems, computer networks, and so forth. We have obtained the closed-form expressions for the steady-state probabilities using recursive technique. Various performance measures such as expected number of customers in the system, average balking rate, average reneging rate, and the average rate of customer loss due to impatience are evaluated. We have also considered a cost optimization problem using particle swarm optimization and quadratic fit search method. Numerical results have been discussed which may be helpful to explore the effect of parameters on performance measures. The technique used in this paper can be applied to analyze more complex models such as $M A P / M / 1 / N$ and $M / M / c / N$ multiple working vacations queues with balking and reneging which are left for future investigations.

\section{References}

[1] P. Liao, "Optimal staffing policy for queueing systems with cyclic demands: waiting cost approach," in Proceedings of the 18th Annual Conference of the Production and Operations Management Society (POMS '07), Dallas, Tex, USA, 2007.

[2] F. A. Haight, "Queueing with balking," Biometrika, vol. 44, pp. 360-369, 1957.

[3] F. A. Haight, "Queueing with reneging," Metrika, vol. 2, no. 1, pp. 186-197, 1959.

[4] C. J. Ancker Jr. and A. V. Gafarian, "Some queueing problems with balking and reneging: I," Operations Research, vol. 11, no. 1, pp. 88-100, 1963.

[5] C. J. Ancker Jr. and A. V. Gafarian, "Some queueing problems with balking and reneging: II," Operations Research, vol. 11, no. 6, pp. 928-937, 1963.

[6] M. O. Abou-El-Ata, "The state-dependent queue: M/M/1/N with reneging and general balk functions," Microelectronics Reliability, vol. 31, no. 5, pp. 1001-1007, 1991. 
[7] M. O. Abou-El-Ata and A. I. Shawky, "The single-server Markovian overflow queue with balking, reneging and an additional server for longer queues," Microelectronics Reliability, vol. 32, no. 10, pp. 1389-1394, 1992.

[8] H. W. Chia and K. Jau-Chaun, "Computational algorithm and parameter optimization for a multi-server system with unreliable servers and impatient customers," Journal of Computational and Applied Mathematics, vol. 235, no. 3, pp. 547-562, 2010.

[9] A. Choudhury and P. Medhi, "Some aspects of balking and reneging in finite buffer queues," RAIRO-Operations Research, vol. 45, no. 3, pp. 223-240, 2011.

[10] K. Rakesh and K. S. Sumeet, "An M/M/1/N queueing model with retention of reneged customers and balking," American Journal of Operational Research, vol. 2, no. 1, pp. 1-5, 2012.

[11] N. Tian and Z. G. Zhang, Vacation Queueing Models: Theory and Applications, Springer, New York, NY, USA, 2006.

[12] J. C. Ke, C. H. Wu, and Z. G. Zhang, "Recent developments in vacation queueing models: a short survey," International Journal of Operations Research, vol. 7, pp. 3-8, 2010.

[13] D. Yue, Y. Zhang, and W. Yue, "Optimal performance analysis of an $\mathrm{M} / \mathrm{M} / \mathrm{1} / \mathrm{N}$ queue system with balking, reneging and server vacation," International Journal of Pure and Applied Mathematics, vol. 28, pp. 101-115, 2006.

[14] S. Vikas and P. Deepali, "Performance analysis of state dependent bulk service queue with balking, reneging and server vacation," International Journal of Operational Research Nepal, vol. 1, pp. 61-69, 2012.

[15] L. D. Servi and S. G. Finn, "M/M/1 queues with working vacations (M/M/1/WV)," Performance Evaluation, vol. 50, no. 1, pp. 41-52, 2002.

[16] P. Vijaya Laxmi, V. Goswami, and K. Jyothsna, "Analysis of finite buffer Markovian queue with balking, reneging and working vacations," International Journal of Strategic Decision Sciences, vol. 4, no. 1, pp. 1-24, 2013.

[17] J. Li and N. Tian, "The M/M/1 queue with working vacations and vacation interruptions," Journal of Systems Science and Systems Engineering, vol. 16, no. 1, pp. 121-127, 2007.

[18] Y. Baba, "The M/PH/1 queue with working vacations and vacation interruption," Journal of Systems Science and Systems Engineering, vol. 19, no. 4, pp. 496-503, 2010.

[19] M. Zhang and Z. Hou, "Performance analysis of M/G/1 queue with working vacations and vacation interruption," Journal of Computational and Applied Mathematics, vol. 234, no. 10, pp. 2977-2985, 2010.

[20] Z. Zhang and $\mathrm{X} . \mathrm{Xu}$, "Analysis for the $\mathrm{M} / \mathrm{M} / 1$ queue with multiple working vacations and $N$-policy," International Journal of Information and Management Sciences, vol. 19, no. 3, pp. 495506, 2008.

[21] J. Kennedy and R. Eberhart, "Particle swarm optimization," in Proceedings of the IEEE International Conference on Neural Networks, vol. 4, pp. 1942-1948, Canberra, Australia, December 1995.

[22] Y. Shi and R. C. Eberhart, "Parameter selection in particle swarm optimization," in Proceedings of the 7th Annual Conference on Evolutionary Programming, pp. 591-600, Springer, 1998.

[23] B. Qinghai, "Analysis of particle swarm optimization algorithm," Computer and Information Science, vol. 3, pp. 180-184, 2010.

[24] S. S. Rao, Engineering Optimization: Theory and Practice, John Wiley \& Sons, Hoboken, NJ, USA, 2009.

[25] R. L. Rardin, Optimization in Operations Research, Prentice Hall, Upper Saddle River, NJ, USA, 1997. 


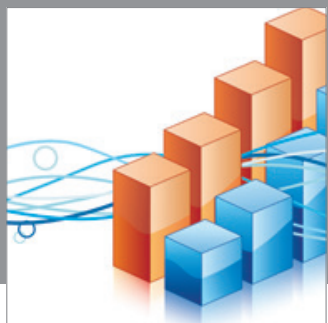

Advances in

Operations Research

mansans

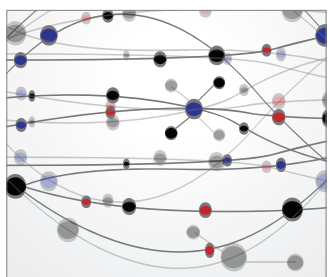

The Scientific World Journal
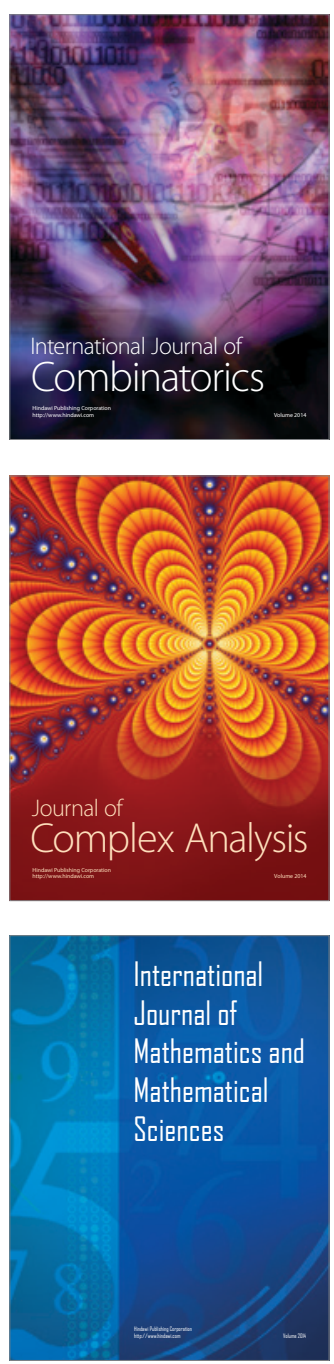
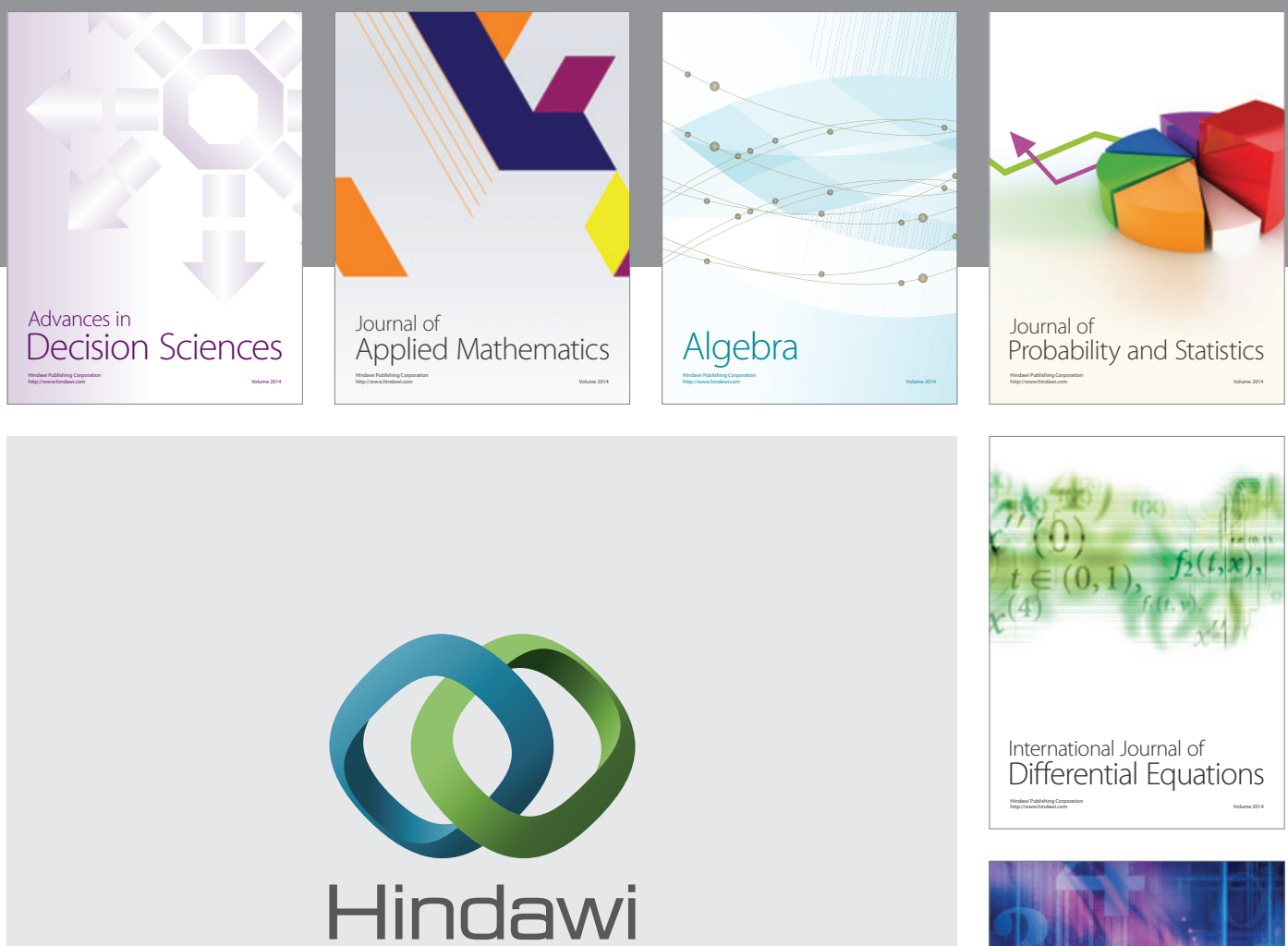

Submit your manuscripts at http://www.hindawi.com
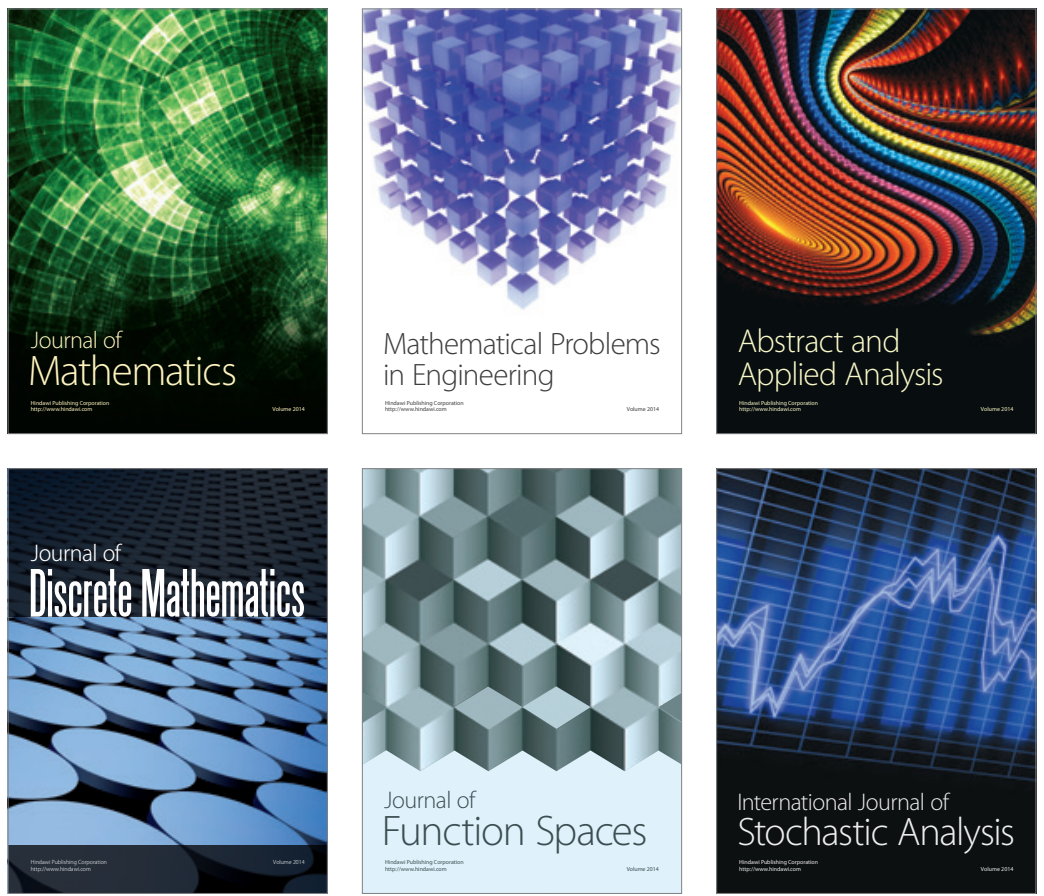

Journal of

Function Spaces

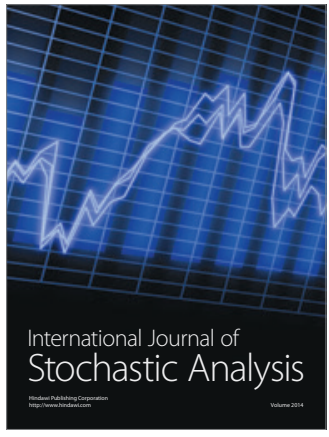

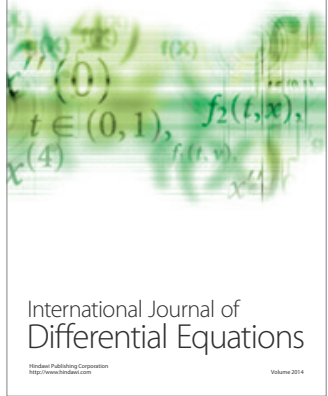
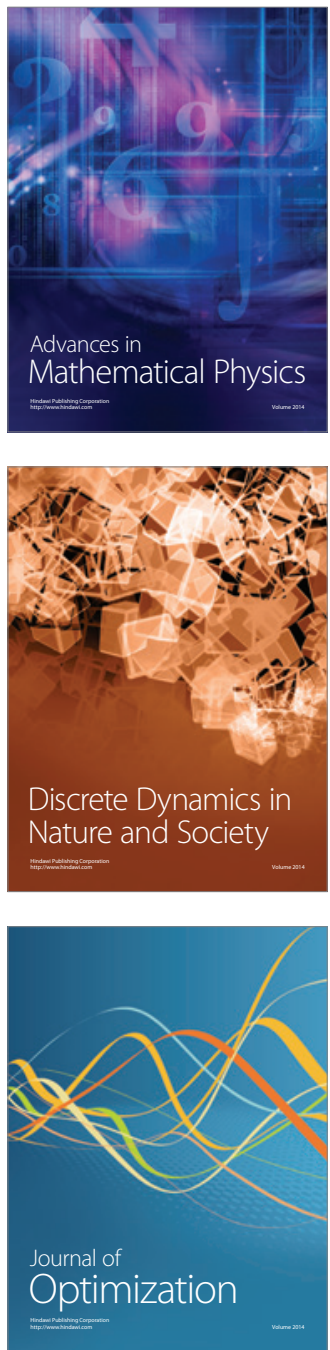